\title{
Flow characteristics of metallic powder grains for additive manufacturing
}

\author{
Bernhard Peters ${ }^{1, \star}$ and Gabriele Pozzetti ${ }^{1, \star \star}$ \\ ${ }^{1}$ Université du Luxembourg 6, rue Coudenhove-Kalergi L-1359 Luxembourg
}

\begin{abstract}
Directed energy deposition technologies for additive manufacturing such as laser selective melting (SLM) or electron beam melting (EBM) is a fast growing technique mainly due to its flexibility in product design. However, the process is a complex interaction of multi-physics on multiple length scales that are still not entirely understood. A particular challenging task are the flow characteristics of metallic powder ejected as jets from a nozzle and shielded by an inert turbulent gas flow. Therefore, the objective is to describe numerically the complex interaction between turbulent flow and powder grains. In order to include both several physical processes and length scales an Euler-Lagrange technology is applied. Within this framework powder is treated by the Discrete-Element-Method, while gas flow is described by Euler approaches as found in classical Computational Fluid Dynamics (CFD). The described method succeeded in delivering more accuracy and consistency than a standard approach based on the volume averaging technique and therefore, is suited for the solution of problems within an engineering framework.
\end{abstract}

\section{Introduction}

Additive manufacturing, also known as 3D printing, processes synthesise a three- dimensional object by adding successive layers of materials under computer control [1] and is sometimes referred to as the third industrial revolution. In its broad sense additive manufacturing includes processes such as binder jetting, directed energy deposition, material extrusion, material jetting, powder bed fusion, sheet lamination and vat photo-polymerisation that apply a variety of materials such as polymeric materials, biological materials, ceramic materials and metallic materials. For metal-based additive manufacturing methods, the metal is supplied in a powder form, with each particle typically having a diameter of $20-100 \mu \mathrm{m}$. Selective laser melting (SLM) and electron beam melting (EBM) apply metallic powders through one or several nozzles onto the substrate's surface where the powder is fused layer by layer into the substrate through an energy input e.g. laser or electron beam. The entire environment is protected by a shielding gas due to the reactive atmosphere of the fusing particles. Thus, the powder interacts strongly with the the fluid dynamics of the shielding gas, that effects crucially the trajectory of single powder particles and hence, its location of deposition. Numerical approaches developed by He et al. [2], Peyre et al. [3], Wen et al. [4] and Qi et al. [5] describe the fusion process, however, neglect the transport of powder between nozzles and substrate. Therefore, the objective of the current contribution is to describe numerically the complex process of powder deposition on a substrate.

\footnotetext{
$\star$ e-mail: bernhard.peters@uni.lu
}

${ }^{\star}$ e-mail: gabriele.pozzetti@uni.lu

\section{Numerical Approach}

The Discrete Element Method (DEM), also called a Distinct Element Method, is probably the most often applied numerical approach to track the trajectories of particles in a system. Thus, DEM is a widely accepted and effective method to address engineering problems in granular and discontinuous materials, especially in granular flows, rock mechanics, and powder mechanics. Pioneering work in this domain has been carried out by Cundall [6], Haff [7], Herrmann [8] and Walton [9]. The volume of Allen and Tildesley [10] is perceived as a standard reference for this field.

\subsection{Extended Discrete Element Method}

The Extended Discrete Element Method considers each particle of an ensemble as an individual entity with motion attached to it. The motion module of the Extended Discrete Element Method (XDEM) handles a sufficient number of geometric shapes that are believed to cover a large range of engineering applications. The exchange of data between continuous and discrete solutions requires careful coordination and a complex feed-back loop so that the coupled analysis converges to an accurate solution. This is performed by coupling algorithms between the Discrete Element Method to Computational Fluid Dynamics (CFD) [11]. For a more detailed review the reader is referred to Peters [12-15]. Hence, an ensemble of discrete and moving particles offers the highest potential to describe transport processes that is characterised by the motion of a rigid body through six degrees of freedom for translation along the three directions in space and rotation about the centreof-mass. 
By describing the degrees of freedom for each particle its motion is entirely determined. Newton's Second Law for conservation of linear and angular momentum describe position and orientation of a particle $i$ as follows:

$$
\begin{aligned}
m_{i} \frac{d^{2} \vec{r}_{i}}{d t^{2}} & =\sum_{i=1}^{N} \vec{F}_{i j}\left(\vec{r}_{j}, \vec{v}_{j}, \vec{\phi}_{j}, \vec{\omega}_{j}\right)+\vec{F}_{\text {extern }} \\
\bar{I}_{i} \frac{d^{2} \vec{\phi}_{i}}{d t^{2}} & =\sum_{i=1}^{N} \vec{M}_{i j}\left(\vec{r}_{j}, \vec{v}_{j}, \vec{\phi}_{j}, \vec{\omega}_{j}\right)+\vec{M}_{\text {extern }}
\end{aligned}
$$

where $\vec{F}_{i j}\left(\vec{r}_{j}, \vec{v}_{j}, \vec{\phi}_{j}, \vec{\omega}_{j}\right)$ and $\vec{M}_{i j}\left(\vec{r}_{j}, \vec{v}_{j}, \vec{\phi}_{j}, \vec{\omega}_{j}\right)$ are the forces and torquues acting on a particle $i$ of mass $m_{i}$ and tensor moment of inertia $\bar{I}_{i}$. Both forces and torques depend on position $\vec{r}_{j}$, velocity $\vec{v}_{j}$, orientation $\vec{\phi}_{j}$, and angular velocity $\vec{\omega}_{j}$ of neighbour particles $j$ that undergo impact with particle $i$. The contact forces comprise all forces as a result from material contacts between a particle and its neighbours. Forces may include external forces due to moving grate bars, fluid forces and contact forces between the particles in contact with a bounding wall. This results in a system of coupled non-linear differential equations which usually cannot be solved analytically.

\subsection{Computational Fluid Dynamics}

Contrary to a pure continuous approach, the current concept treats powder as a discrete entity, while the flow of carrier gas is described by classical Computational Fluid Dynamics (CFD). Since the powder grains are tracked by a Lagrangian approach, their positions are known and thus, the corresponding CFD cell that contains them. Hence, powder grains present in a CFD cell occupy a certain volume meaning introducing a void fraction for the relevant transient differential conservation equations e.g. mass and momentum. Therefore, the flow is treated as a flow in porous media that introduces the porosity concept into the conservation equations. Hence, the turbulent flow (Re 2700) is resolved by a Large Eddy Simulation (LES), that exchanges momentum with the powder grains. This coupling in conjunction with flow predictions is handled by an extended and advanced solver based on the OpenFOAM open-source framework.

\section{Results}

As mentioned-above transport of metallic powder from nozzles to the substrate within a shielding gas atmosphere was predicted by a software framework for advanced multi-physics simulation technology. The set-up of the geometry as depicted in figure 1 includes a central inlet for inert gas, through which also optical access for a laser is provided.

A further ring is arranged concentric to the optical access and serves as location for four nozzles. They inject the metallic powder into the gas phase, that is shielded by an inert gas. It is injected over a conical outer ring and is intended to create an inert atmosphere and to protect the

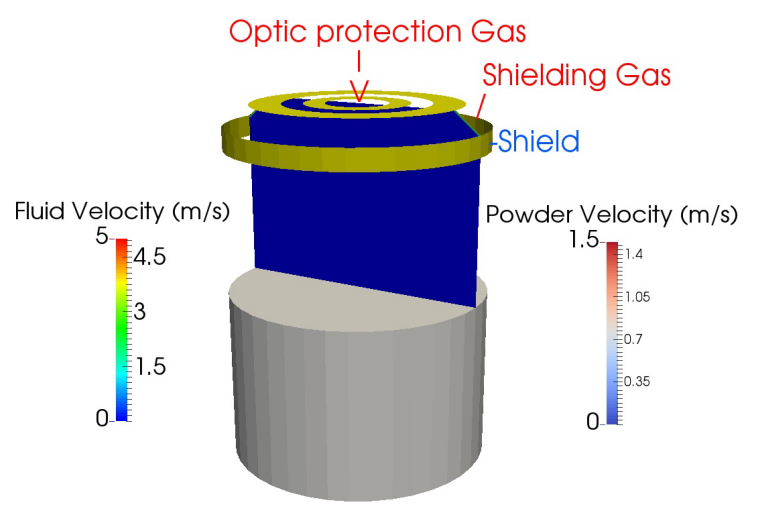

Figure 1: Geometrical set-up of the flow domain for gas and metallic powder

entire environment from entering of oxygen. The shielding gas is redirected radially and outwards due to its direction of the inlet velocity towards the centre axis of the laser. Hence, the velocity field resembles closely a toruslike structure shown in figure 2.

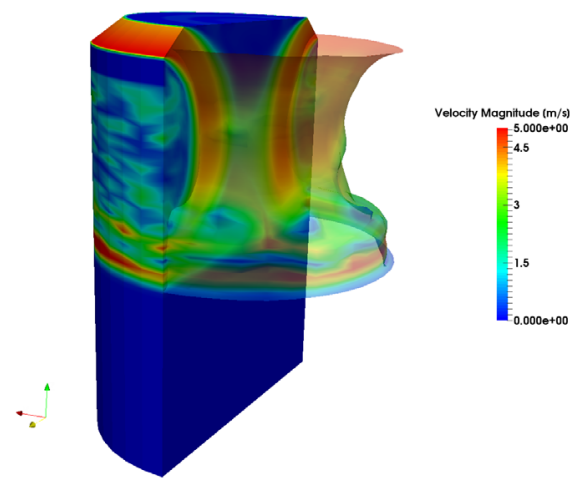

Figure 2: Centre cut through the velocity field with an isosurface of pressure coloured with the velocity magnitude

The iso-surface in figure 2 clearly indicates the spatially varying velocity distribution that undergoes fluctuation over a velocity range of $\sim 5 \mathrm{~m} / \mathrm{s}$. Furthermore, the injected shielding gas directed towards the centre axis of the cylindrical arrangement is redirected radially, that requires radially dependent pressure field. It also is subject to strong spatial variations as indicated by the geometry of the iso-surface. These fluctuations in both velocity and pressure are also strongly reflected by the velocity distribution on the circumferential surface of the cylindrical geometry as depicted in figure 3.

Figure 3 highlights the typical steak-like structure of this class of flow configurations. Additionally, the velocity field shows significant temporal characteristics indicating a swirling flow about the centre axis. Similar to the velocity field in figure 2 , the radial velocity undergoes equally strong fluctuations of $\sim 5 \mathrm{~m} / \mathrm{s}$. Both velocity and pressure field have a significant impact on the trajectories of the 


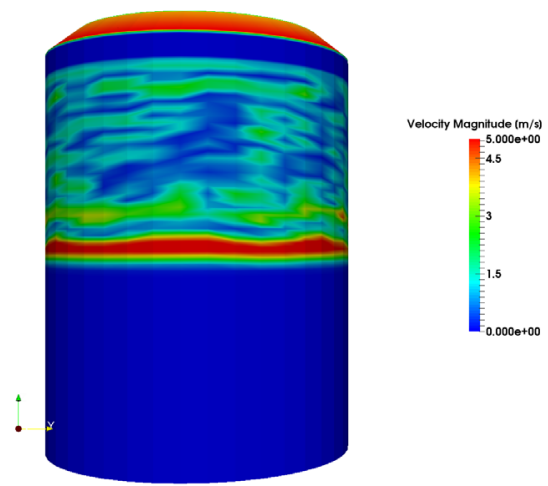

Figure 3: Velocity distribution on the cylindrical circumference

metallic powder that is shown in the sequence of figures $4 \mathrm{a}$ to $4 \mathrm{c}$.

Metallic powder with its size distribution in the range of $\mu m$ has a low inertia and therefore, is bound to follow the gas velocity profiles e.g. streamlines without mayor deviations. thus, the particles follow the velocity contours from the start of injection from the nozzles as depicted in figure $4 \mathrm{a}$. Furthermore, figure $4 \mathrm{a}$ shows also the developing velocity field with a velocity tip penetrating the inert gas atmosphere. Particles are injected almost horizontally with a minor inclination only which requires them to be a radially accelerated to change their direction of the trajectory which can be observed in figure $4 \mathrm{~b}$. Thus, the direction of the metallic powder has changed from an almost horizontal injection direction to a downward flow meaning. The four jets of metallic powder very much focus to the centre area of the laser which is caused by the almost fully developed flow profile of the gas. An inclined injection through a conical area at the inlet generates also a strongly converging flow to the centre axis that the powder follows due to its small inertia as depicted in figure $4 \mathrm{~b}$. The following figure 5 depicts a magnified illustration of the powder grains coloured with their velocity magnitude (powder grains are enlarged for improved visibility and therefore, partially overlap) and the jet closely folowing the streamlines of the carrier gas.

However, the presence of a surface of the work piece, that cannot be penetrated by the gas, induces a diverging flow pattern as shown in figure 4c. Since the flow leaves the geometry over the circumferential area, the direction of the flow has to change again to an outward radial direction as already depicted in figure 3 . Hence, after a converging flow a rapidly diverging gas flow is installed that has also a strong impact on the flow pattern of the metallic powder. As the powder follows very closely the gas velocity, the initially focussed powder jets experience likewise severe diverging flow characteristics. These characteristics generate four detached impact areas at the edge of the melt pool. Hence, no defined focal point exists where the four powder jets penetrate the melt pool that would enable controlled deposition and build-up of the substrate. Additionally, lowest temperatures are expected

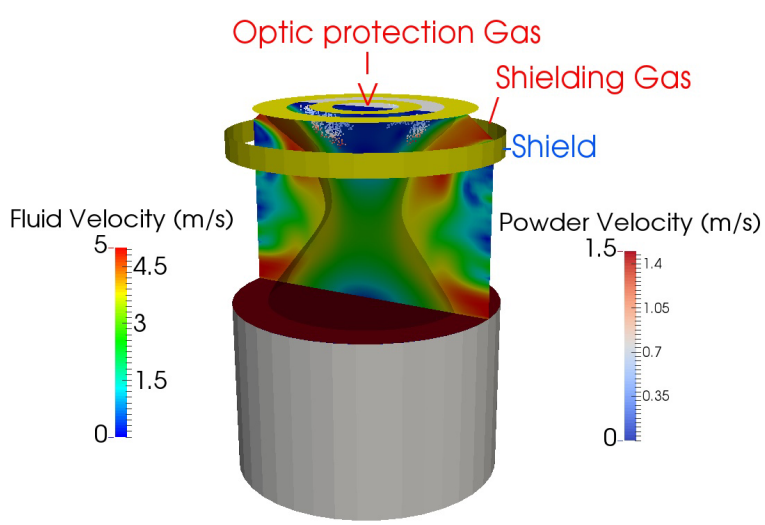

(a) at time: $\mathrm{t}=0.005 \mathrm{~s}$

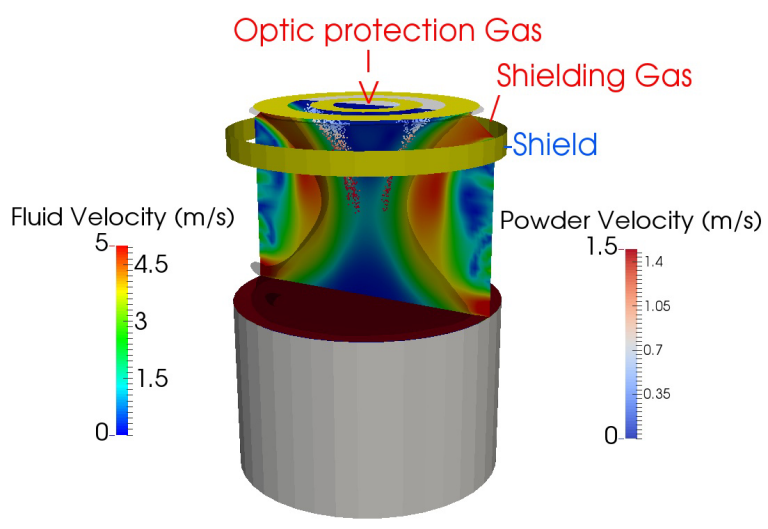

(b) at time: $\mathrm{t}=0.0085$

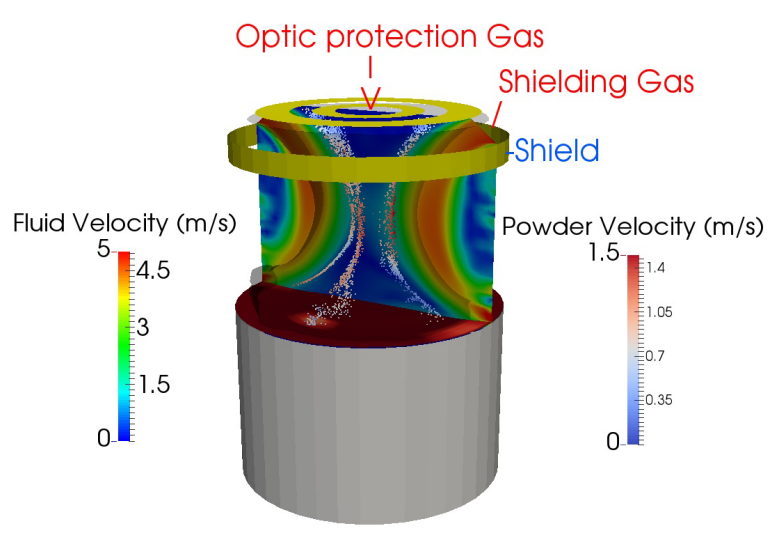

(c) at time: $\mathrm{t}=0.2 \mathrm{~s}$

Figure 4: Centre slice of fluctuating velocity field with instantaneous positions of powder grains

at the edges of the melt pool so that less energy is available for the powder to melt as compared to the centre of the melt pool where the laser energy generates highest temperatures. Reduced temperatures may not be sufficient to melt the biggest powder particles entirely so that they appear as a partially melted inclusion inside the crystal structure of the solidified substrate. Consequently, no homogeneous 


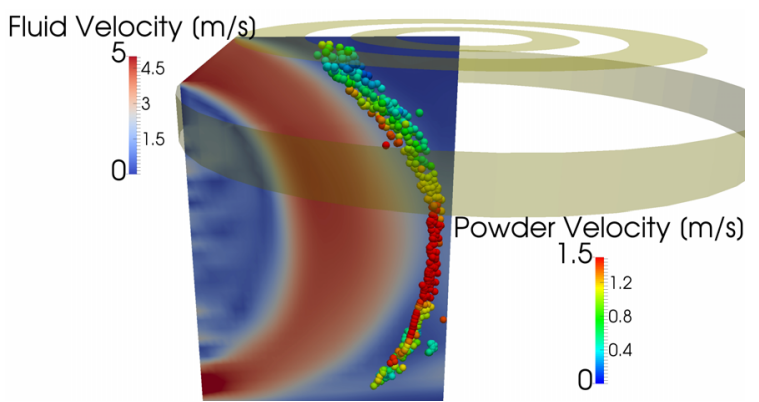

Figure 5: Close-up of a powder jet following closely the streamlines of the carrier gas

crystal structure is obtained, that in addition produces reduced mechanical strength.

\section{Summary}

Within the current study the flow pattern of both shielding gas and four jets of injected metallic powder were investigated numerically. For this purpose, an advanced multi-physics simulation technology was employed to obtain detailed results. The simulation technology is based on the Extended Discrete Element Method (XDEM) that couples discrete with continuous numerical techniques. Hence, the trajectory of the metallic powder grains are resolved with the Discrete Element Method (DEM), while the velocity field of the shielding gas is analysed by classical Computational Fluid Dynamics (CFD). Both phases discrete and continuous exchange momentum, that determines the trajectories of metallic powder to a large extent. Thus, position and velocity of each grain are predicted at all instances of time, that allows tracking the grains until they impact with the melt pool. Identifying the location of deposition of the metallic powder jets is of utmost importance for building up a desired geometry of the substrate. This is very difficult to achieve with experimental devices only, so that numerical techniques are complementing experiments in a favourable way.

\section{References}

[1] J. Excell, The rise of additive manufacturing, retrieved 2016-08-31, https://www . theengineer.co.uk/issues/ 24-may-2010/the-rise-of-additivemanufacturing/

[2] X. He, G. Yu, J. Mazumder, Journal of Physics DApplied Physics 43, 1 (2010)

[3] P. Peyre, P. Aubry, R. Fabbro, R. Neveu, A. Longuet, Journal of Physics D: Applied Physics 41, 025403 (2008)

[4] S.Y. Wen, Y.C. Shin, Journal of Laser Applications 23, 1 (2011)

[5] H. Qi, J. Mazumder, , H. Ki, Journal of Applied Physics 100, 24903 (2006)

[6] P.A. Cundall, O.D.L. Strack, Geotechnique 29, 47 (1979)

[7] P.K. Haff, B.T. Werner, Poweder Techn. 48, 23 (1986)

[8] J.A.C. Gallas, H.J. Herrmann, S. Sokolowski, Phys. Rev. Lett. 69, 1371 (1992)

[9] O. Walton, R. Braun, J. of Rheology 30, 949 (1986)

[10] M.P. Allen, D.J. Tildesley, Computer Simulation of Liquids (Claredon Press Oxford, 1990)

[11] J.H. Ferzinger, M. Peric, Computation Methods for Fluid Dynamics (Springer Verlag, Heidelberg, 1996)

[12] B. Peters, Thermal Conversion of Solid Fuels (WIT Press, Southampton, 2003)

[13] A. Estupinan Donoso, F. Hoffmann, B. Peters, International Review of Mechanical Engineering 7, 328 (2013)

[14] K. Samiei, B. Peters, Chemical Engineering Science 87, 234 (2013)

[15] K. Samiei, G. Berhe, B. Peters, KONA Powder and Particle Journal (2013) 